\title{
Interaction of Lamb Waves with Geometric Discontinuities: An Analytical Approach Compared with FEM
}

\author{
Banibrata Poddar ${ }^{\text {a) }}$ and Victor Giurgiutiu ${ }^{\text {a) }}$ \\ ${ }^{a)}$ Department of Mechanical Engineering, University of South Carolina, USA
}

\begin{abstract}
The non-destructive testing of materials can be conducted by various techniques. Amongst these, method based on ultrasonic waves is one of the most common one. Of these ultrasonic waves Lamb waves are of particular interest for the inspection of large structures for various reasons. Scattering of Lamb waves from flaws has generated a considerable amount of research over last couple of decades. Most of the work has been done using computational tools like Finite Element Methods and experimental technique. In this paper an analytical approach is presented to develop a fundamental understanding of the scattering of Lamb waves from geometric discontinuities in 2 dimensions. We have considered simplest of all geometric discontinuity - a step, as this fundamental understanding can easily be extended to corrosion or crack.
\end{abstract}

\section{INTRODUCTION}

The goal of a structural health monitoring system is to implement processes to detect and characterize damages in engineering structures. Here the damage is defined as changes of material and/or geometric properties of a structure. These systems may use many different physical phenomena to detect and characterize damages. One of these phenomena is elastic wave propagation in thin plate like structures, known as Lamb wave propagation. To develop a SHM (structural health monitoring) system based on Lamb wave propagation we first need to understand how Lamb waves interact with different damage types. In this study we attempted to develop an understanding of how Lamb waves interact with geometric discontinuities using analytical modelling technique. For simplicity we considered only a 2D step type geometric discontinuity as this addresses the fundamental challenge of satisfying the continuous boundary conditions at the location of the discontinuity without getting into a much complicated geometric condition. Also this case can easily be extended to the case of real life damages such as crack or a delamination.

Numerous studies have been done to solve this problem [1-8]. In most of these studies finite element based approach compared with experimental results were used to understand the scattered field. In other studies [3] modal decomposition method was used to predict the scattered wave field. But the continuous boundary condition was scaled down to discrete points across the boundary to satisfy the boundary conditions as it is very challenging to satisfy the continuous boundary condition. In 1982, Gregory et-al $[9,10]$ introduced a method called 'projection method' to satisfy the continuous boundary conditions. This method was also capable of predicting the singularity in stresses in case of a geometric discontinuity [4]. A modified form of projection method was used by Grahn in 2003 [6]. Although this scalar projection method was simpler and able to predict the scattered wave field, the convergence was slow [7]. In 2012 Moreau et-al [7,11] used a vector projection method which had faster convergence and showed promising results.

In this study a form of modal expansion of Lamb modes is used to calculate the scattered wave field. To satisfy the continuous boundary conditions we have used vector project of the boundary conditions to simplify them. Since Rayleigh-Lamb equation has infinite number of complex roots resulting in infinite number of complex Lamb modes, for the sake of convenience we will call this method complex mode expansion with vector projection (CMEP). 


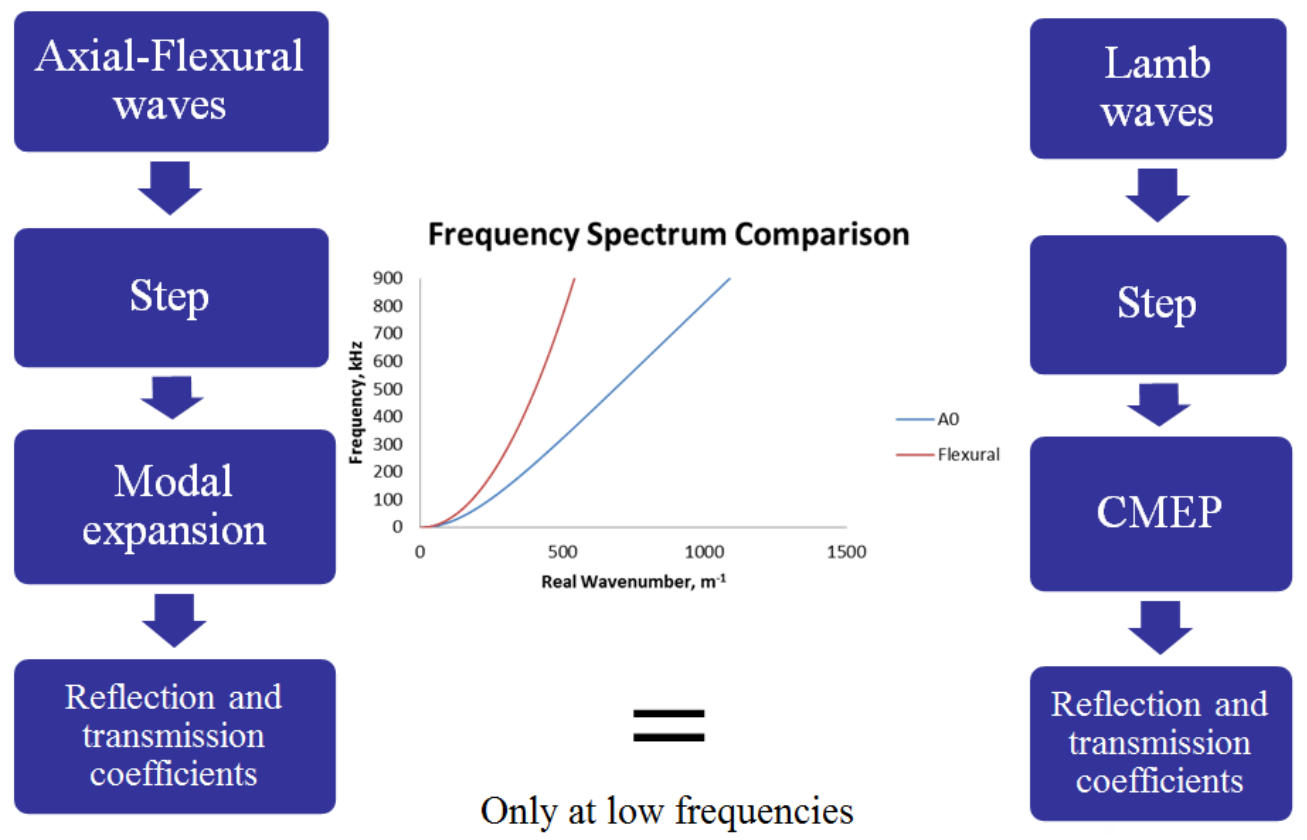

FIGURE 1. Axial flexural waves vs. Lamb waves.

We are going to consider only axial-flexural waves at the step to calculate the scattered wave field. This will serve two purposes. First, it is easier to understand the scattering process using axial-flexural waves compared to Lamb waves which involves complicated mode shapes. Second, the axial-flexural wave based model will act as a benchmark for the Lamb waves based model as axial-flexural waves are low frequency approximation of the Lamb waves. This is illustrated in Fig. 1.

\section{INTERACTION OF AXIAL-FLEXURAL WAVES WITH A STEP}

Let there be a step of depth $d$ along the width in an infinitely long and infinitely wide plate of thickness $h$ at a distance $x=x_{0}$ with eccentricity between the region 1 and region 2 being $a$ as shown in Fig. 2. Also, let us imagine that there is an axial 1D wave and flexural 1D wave travelling in +ve direction in the region 1. Let us define these waves considering the origin at $x=0$ as,

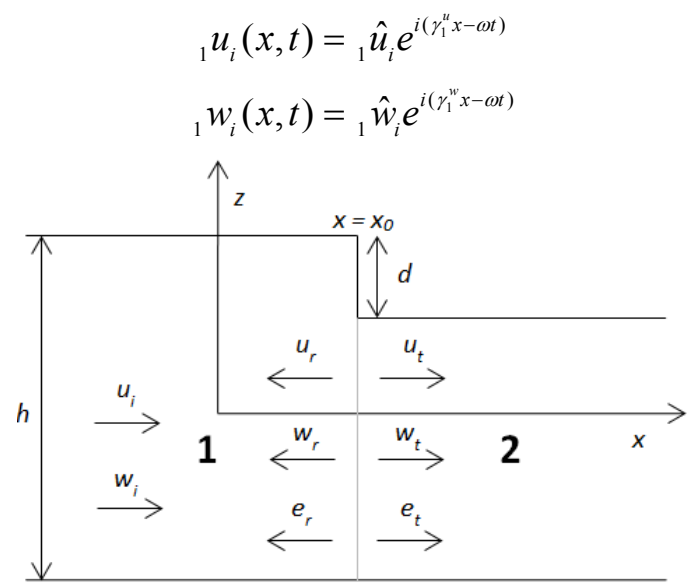

FIGURE 2. Illustration of the step and interacting 1D waves.

After interacting with the step the waves result in a scatter field expressed in terms of all possible modes including the evanescent modes. The scatter field is expressed as, 


$$
\begin{array}{ll}
{ }_{1} u_{r}(x, t)={ }_{1} \hat{u}_{r} e^{i\left[-\gamma_{1}^{u}\left(x-x_{0}\right)-\omega t\right]} & { }_{2} u_{t}(x, t)={ }_{2} \hat{u}_{t} e^{i\left[\gamma_{2}^{u}\left(x-x_{0}\right)-\omega t\right]} \\
{ }_{1} w_{r}(x, t)={ }_{1} \hat{w}_{r} e^{i\left[-\gamma_{1}^{w}\left(x-x_{0}\right)-\omega t\right]} & { }_{2} w_{t}(x, t)={ }_{2} \hat{w}_{t} e^{i\left[\gamma_{2}^{w}\left(x-x_{0}\right)-\omega t\right]} \\
{ }_{1} e_{r}(x, t)={ }_{1} \hat{e}_{r} e^{\gamma_{1}^{w}\left(x-x_{0}\right)-i \omega t} & { }_{2} e_{t}(x, t)={ }_{2} \hat{e}_{t} e^{-\gamma_{2}^{w}\left(x-x_{0}\right)-i \omega t}
\end{array}
$$

where $\mathrm{t}$ stands for transmitted wave and $\mathrm{r}$ stands for reflected waves. The scattered waves have unknown complex amplitude which we need to obtain by applying boundary conditions. The boundary conditions applied at the step are continuity of slope and displacements with force and moment balance at the neutral axis (Fig. 3).

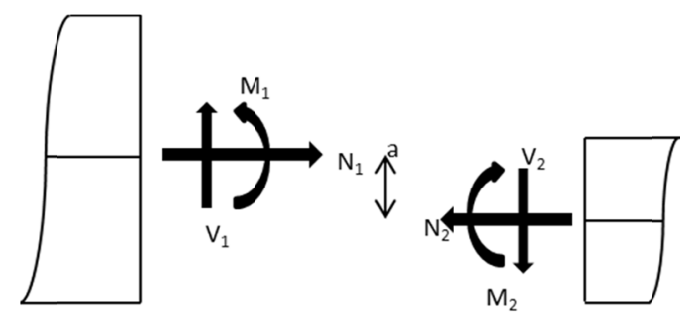

FIGURE 3. Boundary conditions at the step.

This leads us to a set of linear equations which can be expressed as,

$$
\begin{aligned}
& \text { where, }[A]=\left[\begin{array}{cccccc}
0 & -1 & -1 & 0 & 1 & 1 \\
0 & i \gamma_{1}^{w} & -\gamma_{1}^{w} & 0 & i \gamma_{2}^{w} & -\gamma_{2}^{w} \\
-1 & i a \gamma_{1}^{w} & -a \gamma_{1}^{w} & 1 & 0 & 0 \\
E_{1} A_{1} \gamma_{1}^{u} & 0 & 0 & E_{2} A_{2} \gamma_{2}^{u} & 0 & 0 \\
0 & i E_{1} I_{1}\left(\gamma_{1}^{w}\right)^{3} & E_{1} I_{1}\left(\gamma_{1}^{w}\right)^{3} & 0 & i E_{2} I_{2}\left(\gamma_{2}^{w}\right)^{3} & E_{2} I_{2}\left(\gamma_{2}^{w}\right)^{3} \\
0 & -E_{1} I_{1}\left(\gamma_{1}^{w}\right)^{2} & E_{1} I_{1}\left(\gamma_{1}^{w}\right)^{2} & -i a E_{2} A_{2} \gamma_{2}^{u} & E_{2} I_{2}\left(\gamma_{2}^{w}\right)^{2} & -E_{2} I_{2}\left(\gamma_{2}^{w}\right)^{2}
\end{array}\right],\left\{\begin{array}{c}
x_{1} \\
\hat{w}_{r} \\
\hat{w}_{r} \\
\hat{e}_{r} \\
\hat{u}_{t} \\
\hat{w}_{t} \\
\hat{w}_{t} \\
\hat{e}_{t}
\end{array}\right] \begin{array}{c}
\hat{u}_{2} \\
\text { and }
\end{array}
\end{aligned}
$$

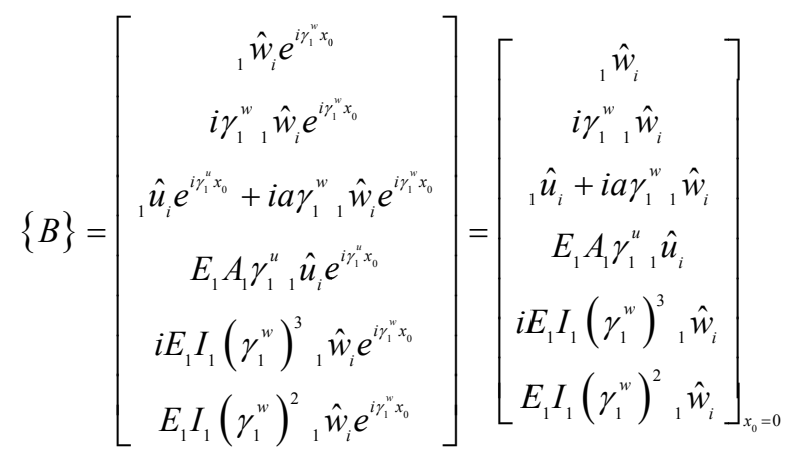

which after solving for $\{X\}$ with $u_{i}=1$ and $w_{i}=0$ we get, 


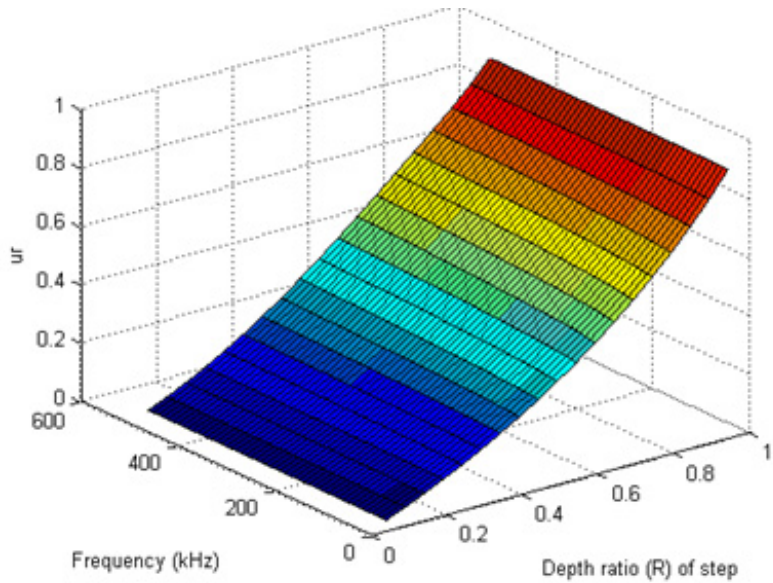

(a)

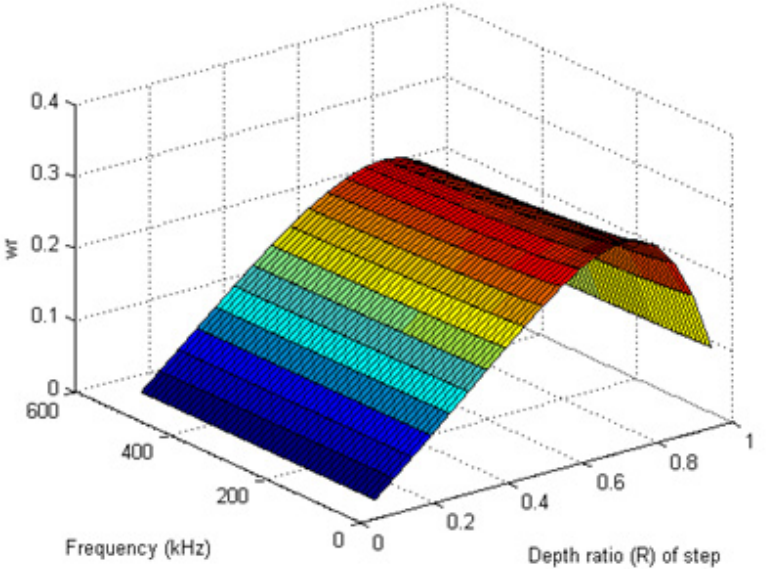

(c)

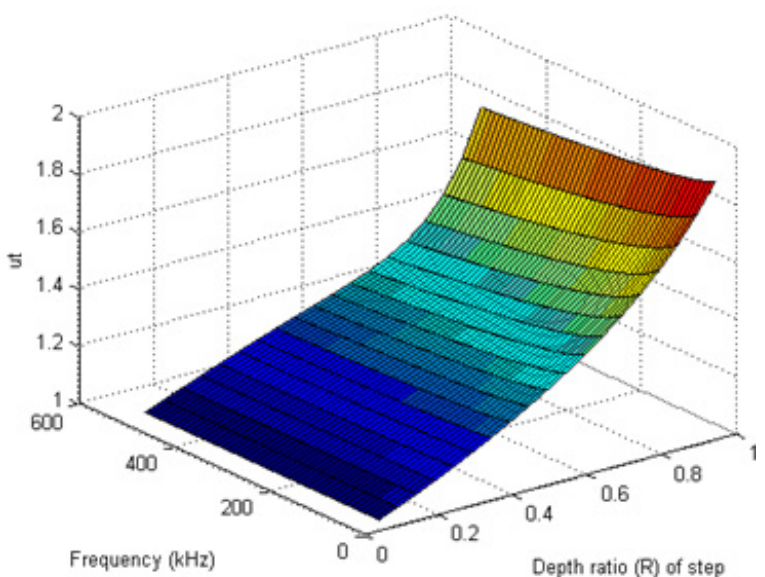

(b)

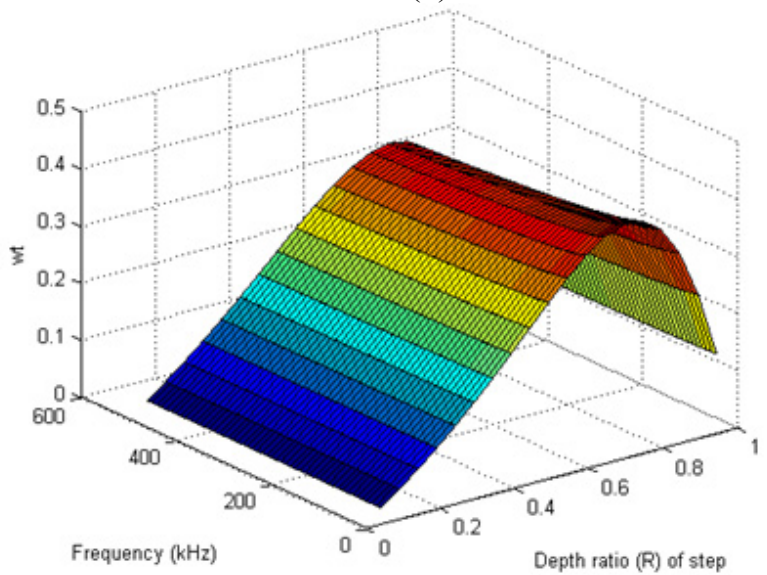

(d)

FIGURE 4. (a) Amplitude of reflected axial wave, (b) amplitude of transmitted axial wave, (c) amplitude of reflected flexural wave and (d) amplitude of transmitted flexural wave.

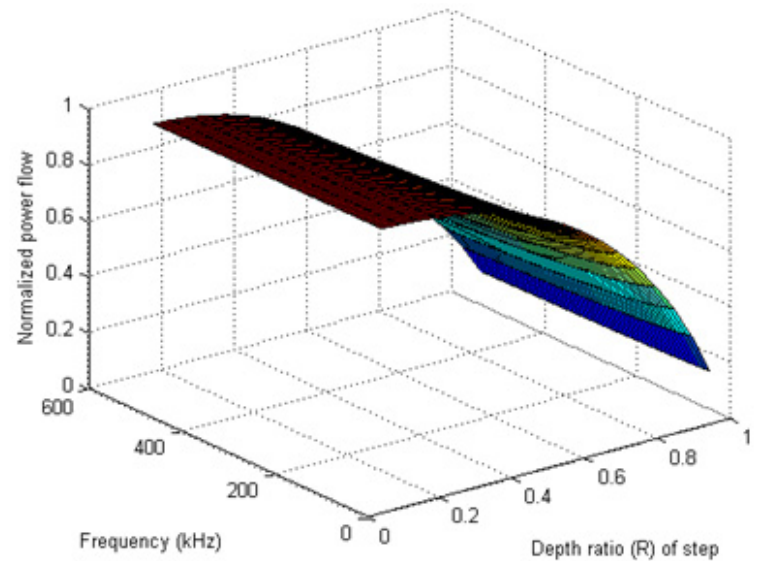

(a)

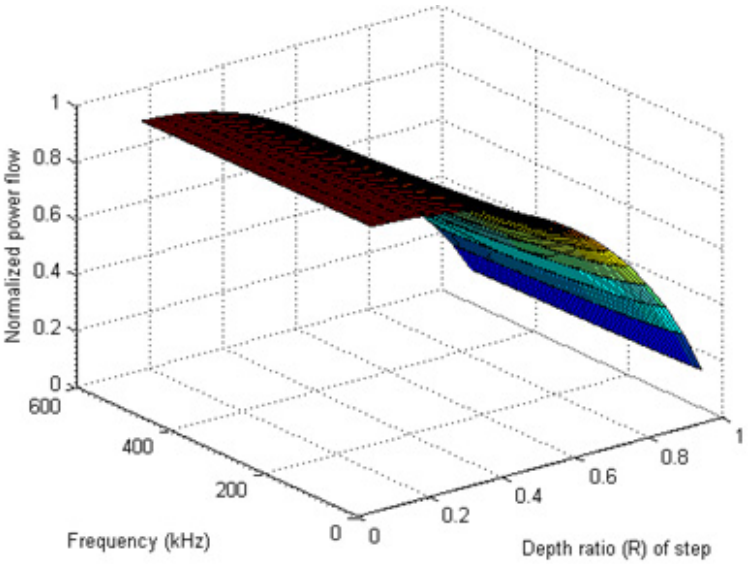

(b)

FIGURE 5. (a) Power flow in region 1 and (b) power flow in region 2.

The power flow through the step is expressed as, 


$$
\begin{aligned}
& \left\langle P_{1}\right\rangle=\omega \frac{E_{1}}{\left(1-v^{2}\right)}\left(\frac{1}{2} A_{1} \gamma_{1}^{u} \operatorname{Re}\left({ }_{1} u_{i 1} \overline{u_{i}}-{ }_{1} u_{r 1} \overline{u_{r}}\right)+I_{1}\left(\gamma_{1}^{w}\right)^{3} \operatorname{Re}\left({ }_{1} w_{i 1} \overline{w_{i}}-{ }_{1} w_{r 1} \overline{w_{r}}\right)\right) \\
& \left\langle P_{2}\right\rangle=\omega \frac{E_{2}}{\left(1-v^{2}\right)}\left(\frac{1}{2} A_{2} \gamma_{2}^{u}{ }_{2} \operatorname{Re}\left({ }_{2} u_{t 2} \overline{u_{t}}\right)+I_{2}\left(\gamma_{1}^{w}\right)^{3} \operatorname{Re}\left({ }_{2} w_{t 2} \overline{w_{t}}\right)\right)
\end{aligned}
$$

In Fig. 5 we can see that the power flow is balanced across the step. From the power expressions we can notice that there is no contribution of the evanescent modes to the power flow. However it is very important to consider them for modal expansion as they are solutions of the governing equation of the wave field. Without considering those the boundary conditions cannot be satisfied.

Therefore we need to find all possible solutions of the Rayleigh-Lamb equation to apply modal expansion for satisfying the boundary conditions.

\section{SOLVING RAYLEIGH-LAMB EQUATION}

Since Rayleigh-Lamb equation is a transcendental equation and does not have closed form solution, we have found the roots of this equation over large domain of complex plane by using recursive iterative algorithms written in MATLAM. The algorithm can converge to root within specified accuracy with certainty. This is important as we need to use correct root of this equation for CMEP.

$$
\frac{\tan \eta_{P} d}{\tan \eta_{S} d}=-\left[\frac{\left(\xi^{2}-\eta_{S}^{2}\right)^{2}}{4 \xi^{2} \eta_{P} \eta_{S}}\right]^{ \pm 1} \quad \eta_{P}^{2}=\frac{\omega^{2}}{c_{P}^{2}}-\xi^{2}, \eta_{S}^{2}=\frac{\omega^{2}}{c_{S}^{2}}-\xi^{2}
$$

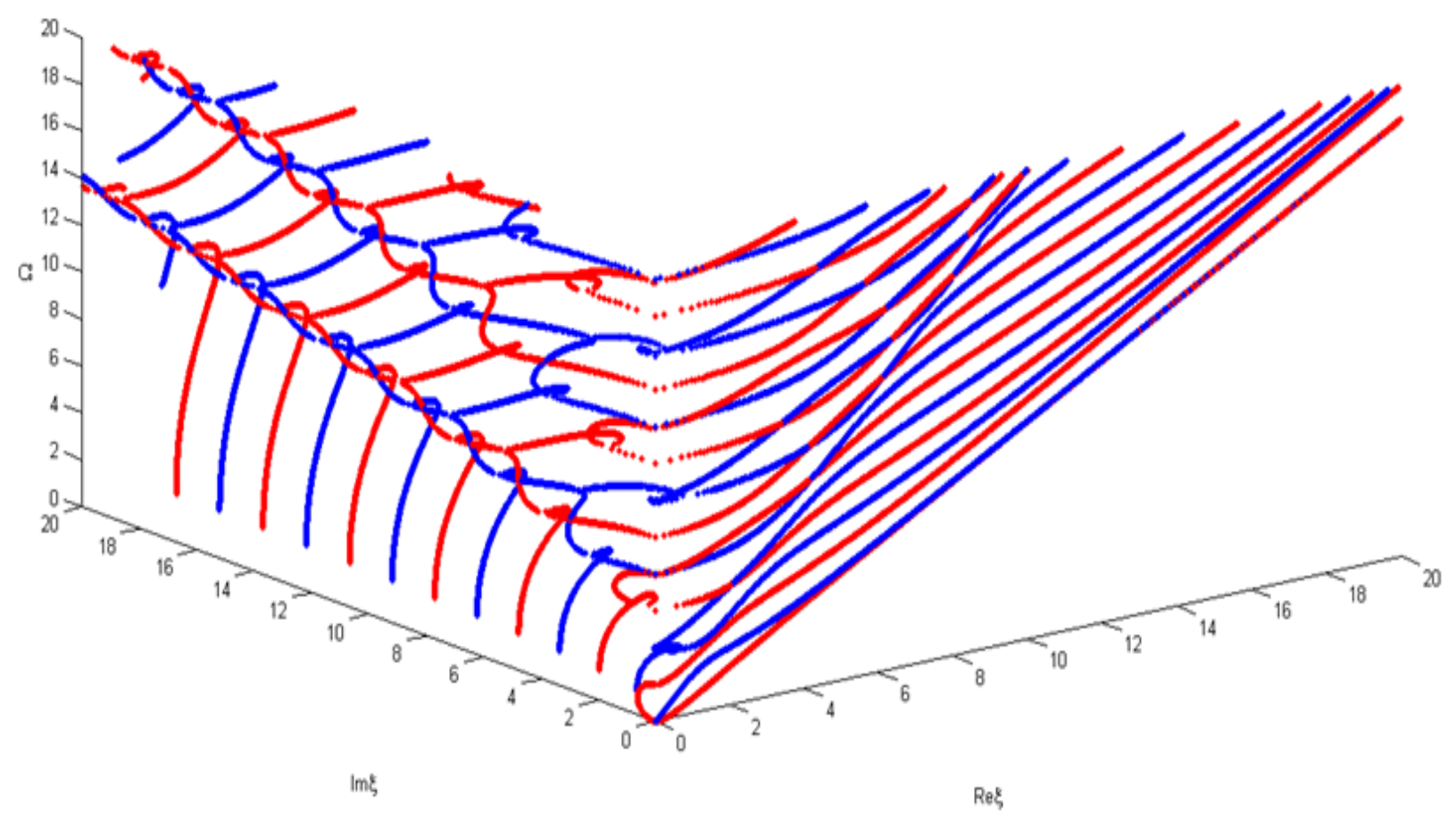

FIGURE 6. Roots of Rayleigh-Lamb equation. Symmetric modes are plotted in blue and antisymmetric modes are plotted in red. 


\section{INTERACTION OF LAMB WAVES WITH STEP}

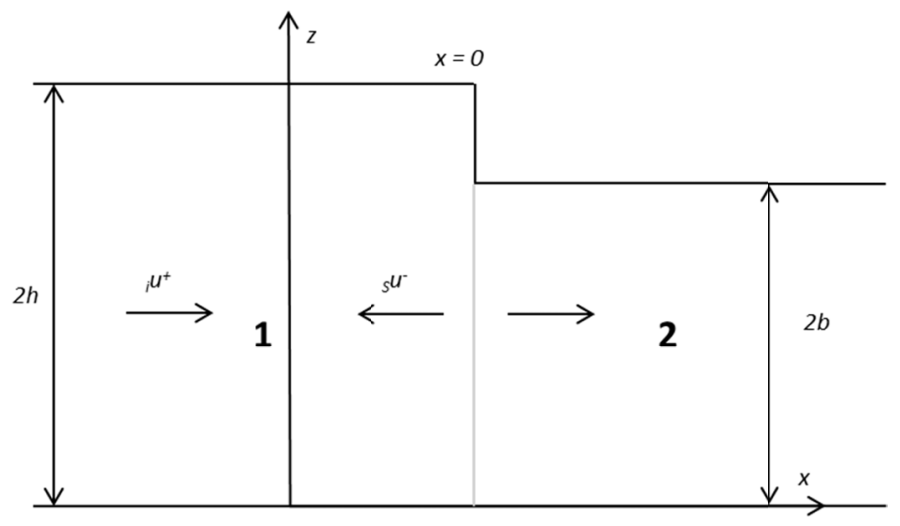

FIGURE 7. Illustration of the step and interacting Lamb waves.

For Lamb waves we consider the incident wave field as,

and the scattered field as,

$$
\begin{aligned}
& \overrightarrow{u_{i}}=\sum_{j=1}^{\infty} C_{j 1}^{+} \overrightarrow{u_{j}} e^{i\left(+\xi_{j} x-\omega t\right)} \\
& \overrightarrow{\sigma_{i}}=\sum_{j=1}^{\infty}{ }_{i} C_{j 1}^{+} \overrightarrow{\sigma_{j}} e^{i\left(+\xi_{j} x-\omega t\right)}
\end{aligned}
$$

$$
\begin{aligned}
& \overrightarrow{u_{s}}=\sum_{j=1}^{\infty}\left({ }_{s} C_{j}^{+} \overrightarrow{u_{j}} e^{i\left(+\xi_{j} x-\omega t\right)}+{ }_{s} C_{j 1}^{-} \overrightarrow{u_{j}} e^{i\left(-\xi_{j} x-\omega t\right)}\right) \\
& \overrightarrow{\sigma_{s}}=\sum_{j=1}^{\infty}\left({ }_{s} C_{j}^{+} \overrightarrow{\sigma_{j}} e^{i\left(+\xi_{j} x-\omega t\right)}+{ }_{s} C_{j 1}^{-} \overrightarrow{\sigma_{j}} e^{i\left(-\xi_{j} x-\omega t\right)}\right)
\end{aligned}
$$

where, subscript " $i$ " stands for incident waves and " $s$ " stands for scattered waves with superscript "+" for waves travelling in $+x$ direction and "-" for waves travelling in $-x$ direction. Also the subscript " $j$ " represents different wave modes.

The boundary conditions applied at the step are displacement boundary condition,

$$
\vec{u}=\vec{u}, \quad 0<z<2 b, \quad x=0
$$

and traction boundary conditions,

$$
\begin{aligned}
& \overrightarrow{{ }_{1} t}=\left\{\begin{array}{ccc}
0, & 2 b<z<2 h, & x=0 \\
\overrightarrow{{ }_{2} t}, & 0<z<2 b, & x=0
\end{array}\right. \\
& \Rightarrow \vec{\sigma}=\left\{\begin{array}{ccc}
0, & 2 b<z<2 h, & x=0 \\
\overrightarrow{{ }_{2} \sigma}, & 0<z<2 b, & x=0
\end{array}\right.
\end{aligned}
$$

We project them onto an appropriate complete orthogonal vector space to remove the $\mathrm{z}$ dependence of the boundary condition. To take advantage of the orthogonality of stress and displacement mode shapes of Lamb wave modes, we choose ${ }_{1} \bar{u}_{j}=\operatorname{conj}\left(\overrightarrow{ }_{1} \vec{u}_{j}\right)$ as the projection vectors for the stress boundary conditions and ${ }_{2} \bar{\sigma}_{j}=\operatorname{conj}\left(\overrightarrow{{ }_{2} \sigma_{j}}\right)$ as the projection vectors for the displacement boundary conditions. The final forms of the boundary conditions independent of $\mathrm{z}$ are, 


$$
\begin{aligned}
& \int_{0}^{2 b}\left(\overrightarrow{{ }_{1} u}-\overrightarrow{{ }_{2} u}\right)_{2} \bar{\sigma}_{j} d z=0 \Rightarrow\left\langle\left(\overrightarrow{{ }_{2} u_{S}}-\overrightarrow{{ }_{1} u_{S}}\right),{ }_{2} \bar{\sigma}_{j}\right\rangle=\left\langle\overrightarrow{ }_{1}{ }_{i},{ }_{2} \bar{\sigma}_{j}\right\rangle ; \quad x=0 \\
& \int_{0}^{2 h} \overrightarrow{ }_{1}{ }_{1} \bar{u}_{j} d z=\int_{0}^{2 b} \overrightarrow{ }_{2}{ }_{1} \bar{u}_{j} d z \Rightarrow\left\langle\left(\overrightarrow{{ }_{2} \sigma_{S}}-\overrightarrow{{ }_{1} \sigma_{S}}\right),{ }_{1} \bar{u}_{j}\right\rangle=\left\langle\overrightarrow{{ }_{1}},{ }_{1} \bar{u}_{j}\right\rangle ; \quad x=0
\end{aligned}
$$

This leads us to the set of linear equations expressed as,

$$
[A]_{2 j \times 2 j}\{C\}_{2 j \times 1}=\{B\}_{2 j \times 1}
$$

For numerical results we need to determine the maximum number of roots of Rayleigh-Lamb equation to be considered which should give us reasonably accurate result. Figure 9 shows the convergence of the modal participation factors of the first three modes of the Lamb waves and we can see that 27 modes are more than enough for the result to converge. Then the above equation can be easily solved using matrix inversion in MATLAB.

\section{Convergence of the reflection and transmission cefficients}

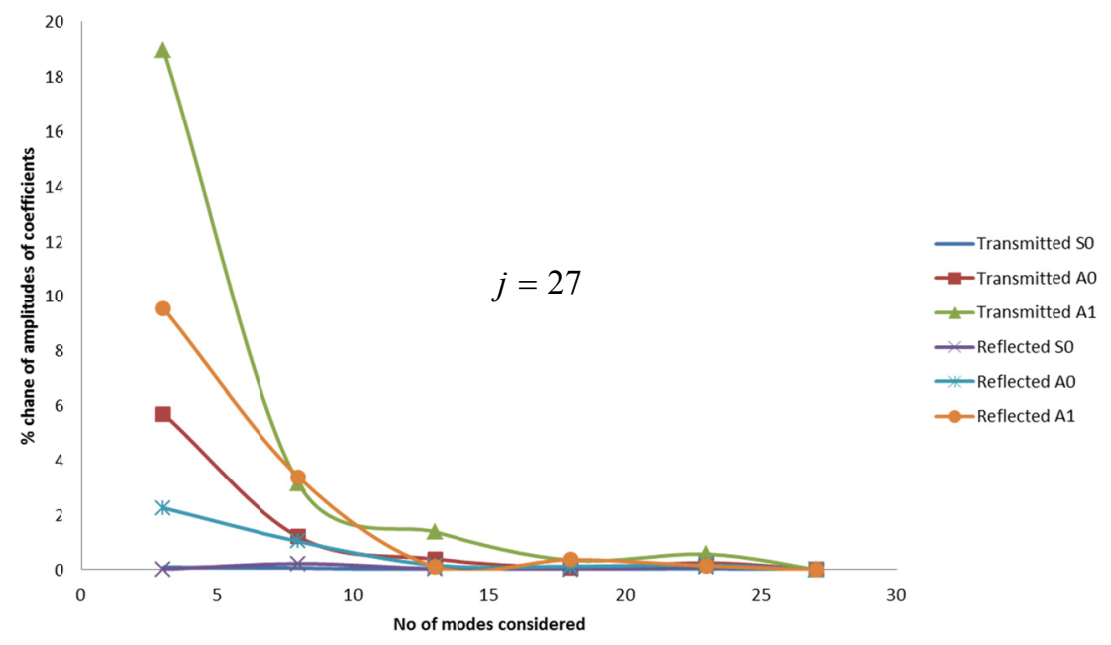

FIGURE 8. Convergence study.

\section{COMPARISON WITH 1D WAVES}

Since axial and flexural waves are the low frequency approximation of the Lamb waves, the scatter field for 1D waves should be same as scatter field of Lamb waves at low frequency. For comparison we have calculated scattered field of the 1D waves and Lamb waves for step size of $1 \mathrm{~mm}$ in a $2 \mathrm{~mm}$ plate with $\mathrm{S}_{0}$ mode as the incident Lamb wave mode.

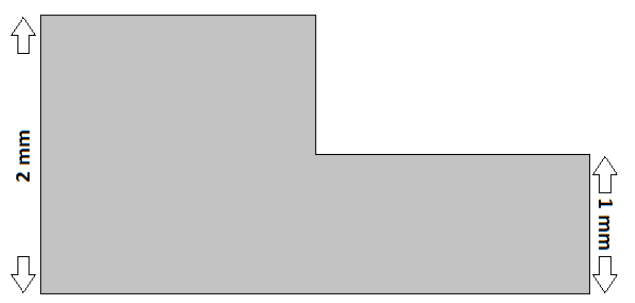

FIGURE 9. Geometry of step considered. 




(a)

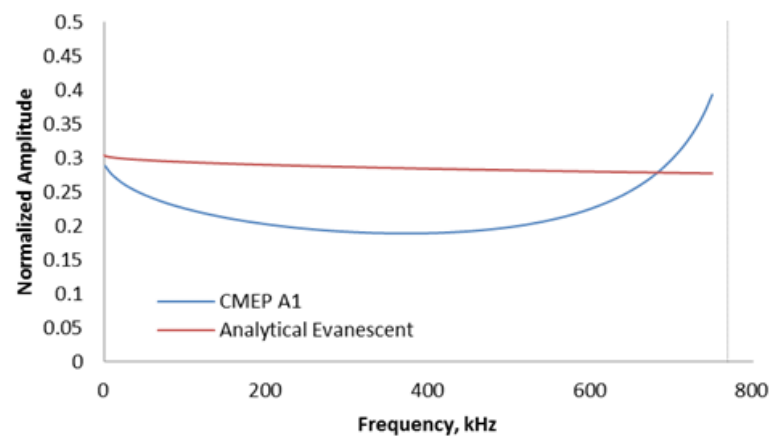

(c)

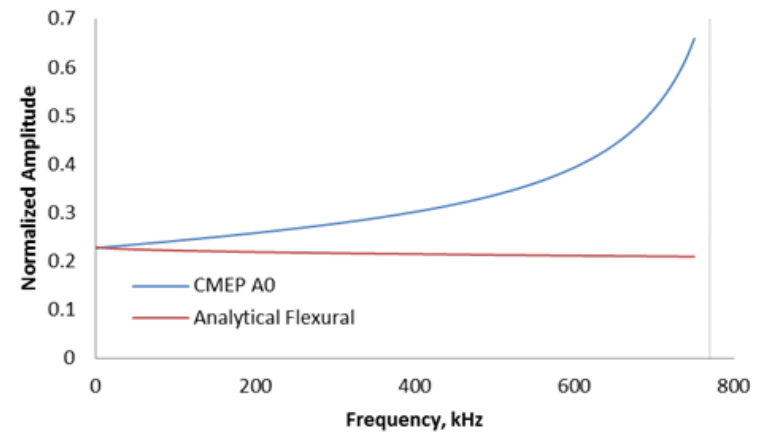

(b)

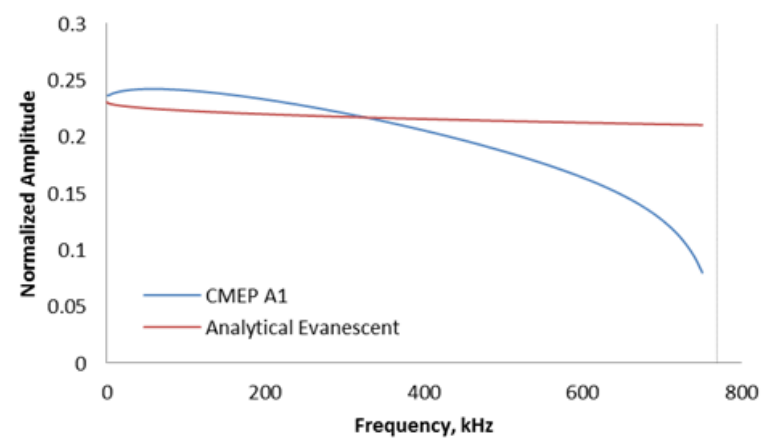

(d)

FIGURE 10. Comparison of y displacement at neutral plane (a), (c) transmitted waves and (b), (d) reflected waves.

\section{FEM MODEL}

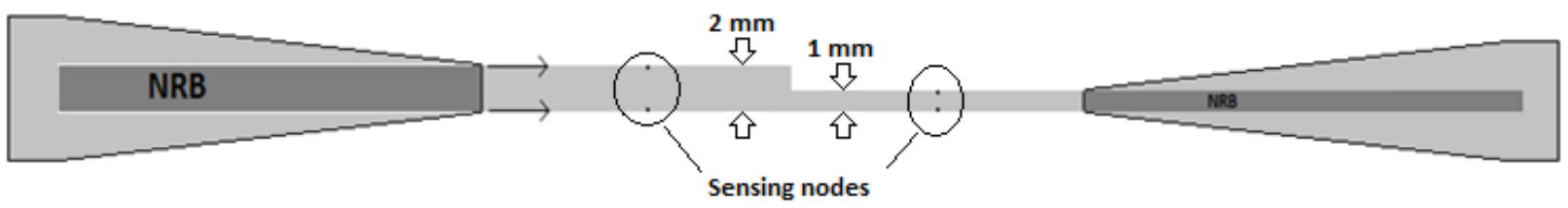

FIGURE 11. A FEM model.

To model interaction of Lamb waves with step we used 2D plane strain model. To obtain the scatter coefficients as a function of frequency we did harmonic analysis. Harmonic analysis normally will produce standing wave field. To get a transient response we introduced non reflective boundary at the edges of the model such that they do not reflect any wave. Therefore doing a harmonic analysis with nonreflecting boundary enabled us to obtain the scatter coefficients as a function of time. The nonreflecting boundary was created using linear spring damper element attached to the surface of the boundaries. The damping constants were varied such that no reflection occurs at the nonreflecting boundary. The simulation was done for incident $\mathrm{S}_{0}$ Lamb wave mode.

From Fig. 12 we can see that the results from FEM and CMEP match perfectly. The minor noise in the FEM data was due to the numerical noise generated by round-off errors. 


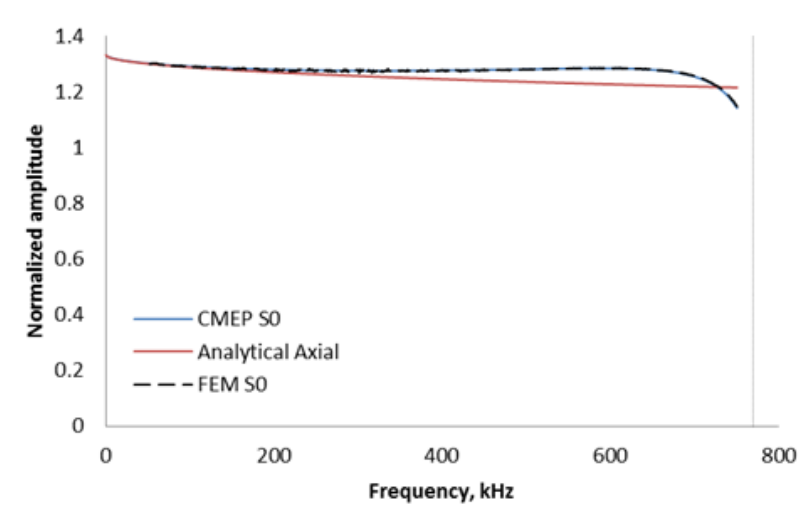

(a)

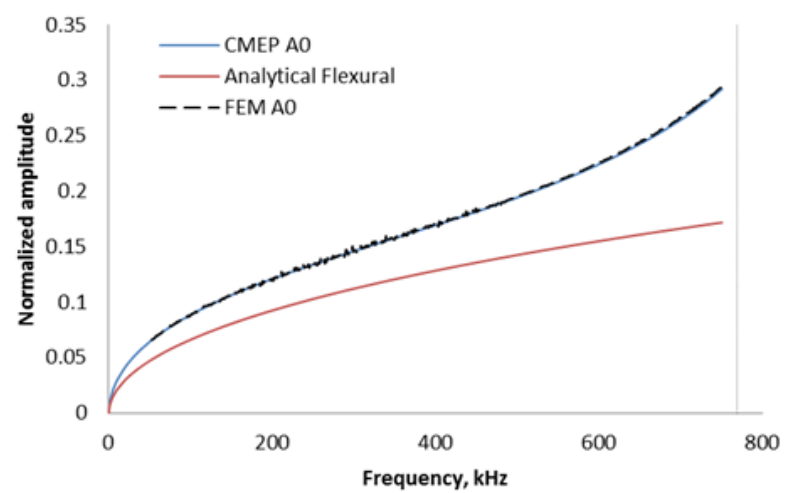

(c)

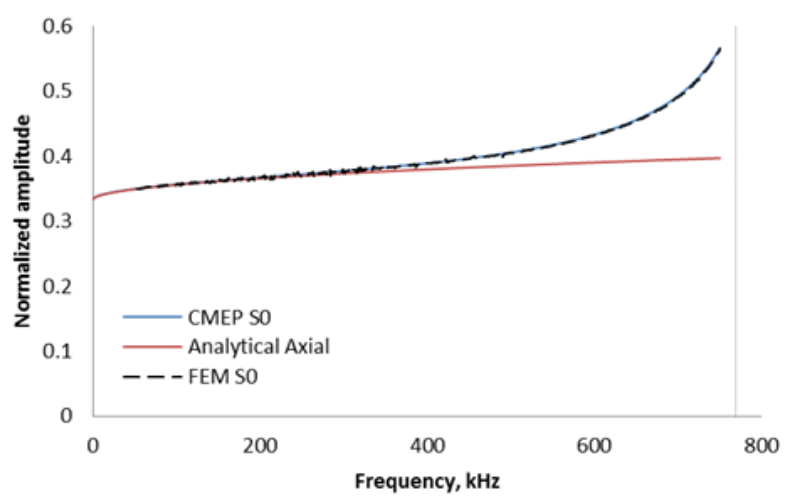

(b)

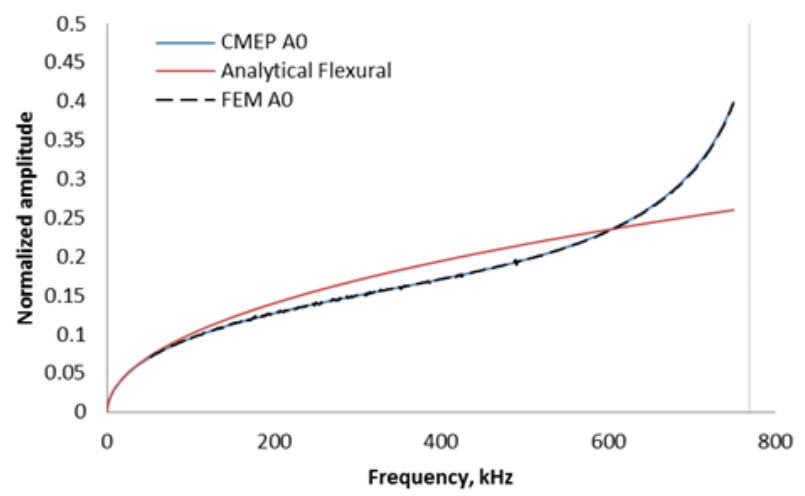

(d)

FIGURE 12. Comparison of top surface $x$ displacement (a), (c) transmitted waves and (b), (d) reflected waves.

\section{SUMMARY AND CONCLUSION}

A robust algorithm was developed to find roots of Rayleigh-Lamb equation over a very complex domain with controlled accuracy. An analytical, CMEP was developed to predict the scatter field of Lamb wave produced by a geometric discontinuity. Along with that a simplified model to predict scattering of $1 \mathrm{D}$ waves from geometric discontinuity was developed mainly to develop better understanding. Both the model agree with each other at low frequency confirming their validity. A FEM model was made to compare and validate CMEP. The numerical model proved CMEP to be an accurate prediction of the scatter field. Vice versa CMEP confirmed the validity of the result of FEM simulation. This also proves that CMEP can be used to check for FEM model validity. CMEP also shows that it is possible to obtain the scatter field of Lamb wave analytically. CMEP can also predict the local field of vibration in terms of nonpropagating Lam wave modes.

The technique of vector project can be extanded for 3D geometric discontinuities and real damages such as cracks and delamination. Therefore CMEP can be extended to model scattering of Lamb waves in 3D geometries without much difficulties.

\section{ACKNOWLEDGMENTS}

Support from office of Naval Research \# N00014-11-1-0271, Dr. Ignacio Perez, Technical Representative and Air Force Office of Scientific Research \#FA9550-11-1-0133, Dr. David Stargel, Program Manager; are thankfully acknowledged. 


\section{REFERENCES}

1. D. N. Alleyne and P. Cawley, "The interaction of Lamb waves with defects," IEEE Trans Ultrason Ferroelectr Freq Control, 39 (3), 381-97 (1992).

2. D. N. Alleyne and P. Cawley, "The effect of discontinuities on the long-range propagation of Lamb waves in pipes," Proc Inst Mech Eng, Part E J Process Mech Eng., 210-217 (1996).

3. M. Castaings, E. Le Clezio, and B. Hosten, "Modal decomposition method for modeling the interaction of Lamb waves with cracks," J Acoust Soc Am., 112 (6), 2567-2582 (2002).

4. M. A. Flores-López and Gregory R. Douglas, "Scattering of Rayleigh-Lamb waves by a surface breaking crack in an elastic plate," J Acoust Soc Am., 119 (4), 2041 (2006).

5. E. V. Glushkov, N. V. Glushkova, and O. N. Lapina, "Diffraction of Normal Modes in Composite and Stepped Elastic Waveguides. J Appl Math Mech., 62 (2), 275-280 (1998).

6. T. Grahn, "Lamb wave scattering from a circular partly through-thickness hole in a plate,". Wave Motion. 37 (1), 63-80 (2003).

7. L. Moreau, M. Caleap, A. Velichko, and P. D. Wilcox, "Scattering of guided waves by flat-bottomed cavities with irregular shapes," Wave Motion, 49 (2), 375-387 (2012).

8. S. Rokhlin, "Diffraction of Lamb waves by a finite crack in an elastic layer," J Acoust Soc Am., 67 (4), 1157-1165 (1980).

9. $\quad$ Gregory R. Douglas and I. Gladwell, "The cantilever beam under tension, bending or flexure at infinity," $J$ Elast. 12 (4), 317-343 (1982).

10. Gregory R. Douglas and I. Gladwell, "The reflection of a symmetric Rayleigh-Lamb wave at the fixed or free edge of a plate," J Elast., 13, 185-206 (1983).

11. L. Moreau, M. Caleap, A. Velichko, and P. D. Wilcox, "Scattering of guided waves by through-thickness cavities with irregular shapes," Wave Motion, 48 (7), 586-602 (2011). 